\title{
SPONTANEOUS SEPTOSTOMY IN TWIN PREGNANCY: A RARE AND DANGEROUS COMPLICATION
}

Chitra Andrew ${ }^{1}$, K. S. Rajeswari ${ }^{2}$

\section{HOW TO CITE THIS ARTICLE:}

Chitra Andrew, K. S. Rajeswari. "Spontaneous Septostomy in Twin Pregnancy: A Rare and Dangerous Complication". Journal of Evolution of Medical and Dental Sciences 2014; Vol. 3, Issue 63, November 20; Page: 13931-13936, DOI: 10.14260/jemds/2014/3862

ABSTRACT: Monochorionic diamniotic twin pregnancies are prone to many complications which are unique to this type of twinning. These complications include twin twin transfusion and selective fetal growth restriction. However a rare complication of twinning is spontaneous septostomy of the dividing membrane. This converts the diamniotic pregnancy into a monoamniotic pregnancy with the added complication of cord entanglement which increases mortality and morbidity. A case report of this condition has been described and a detailed literature review performed.

KEYWORDS: Amniotic Membrane, Monochorionic Diamniotic Twin Pregnancy, Twin transfusion.

INTRODUCTION: Chorionicity and amnionicity influence every aspect of multiple pregnancy and its management. Chorionicity is best seen at the $11-14$ weeks scan during which time the dichorionic diamniotic pregnancy shows a twin peak sign (lambda sign). The finding of a $\mathrm{T}$ sign means the pregnancy is monochorionic and the fetuses share a placenta. These pregnancies have problems which are unique to this type of placentation which include twin - twin transfusion, selective fetal growth restriction and co twin demise leading to hypoxic sequel in the survivor. For these reasons, monochorionic pregnancies have different monitoring protocols. These monitoring protocols are designed to reduce or predict the worst of complications and optimize outcome. Monoamniotic pregnancies are a rare form of monochorionic pregnancy which have the additional complication of entanglement of the umbilical cords leading to sudden intra-uterine death.

CASE REPORT: Mrs. P, a 36 year old lady, primigravida conceived during her third cycle of ovulation induction with Clomiphene after 6 years of infertility. She was a pre-gestational diabetic since two years. She was on metformin 500mg twice daily. Her glycosylated hemoglobin performed at 8 weeks gestation was 7 percent.

She was referred to the fetal medicine unit for a scan at 12 weeks of pregnancy and the scan findings were as follows. The membrane seen between the fetuses with a " $\mathrm{T}$ " insertion, and single anterior placenta was seen. (Fig 1) Both fetuses showed normal liquor in their sacs and fetal activity was present. Fetus A: CRL $-60 \mathrm{~mm}$ NT $-1.7 \mathrm{~mm}$, B: CRL $-58 \mathrm{~mm}$ NT $-1.7 \mathrm{~mm}$. The diagnosis of monochorionic diamniotic twin pregnancy was made. At that point in time, both fetuses showed no significant difference in the crown rump lengths or the nuchal translucency. The plan was to review at 16 weeks for re-evaluation and Doppler. The couple was advised to have the combined biochemical screening test performed the same day.

The couple did not review at 16 weeks but returned at 19 weeks. The combined biochemical screening test result was negative. At this time, normal liquor was seen around both fetuses. (Fig.2)

The fetus A showed an absent bladder and fetus B had large bladder. (Fig. 3) There was a discrepancy in the growth (Fig.4) which was 30\%. Fetus B had a normal flow in the umbilical artery, 


\section{CASE REPORT}

and ductus venosus on Doppler flow. (Fig. 5, 6) Fetus A showed high resistance flow in umbilical artery Doppler.

The scan findings were inconsistent as the fetus with absent bladder and growth restriction with altered flow pattern on Doppler would be consistent with a diagnosis of Twin twin transfusion (Stage 3 Quintero) and would be expected to have oligohydramnios or anhydramnios. This was not demonstrable. The possibility that the fetus would be wrapped in the membranes making it difficult to appreciate the membrane was considered, but the limbs were seen to move freely. No folding of the inter-twin membrane was demonstrable. The other potential differential diagnosis was selective growth restriction, which did not explain the absence of bladder. The patient was in discomfort due to prolonged lying down for the ultrasound examination and was allowed to rest before further scanning.

When she returned, after two hours, the fetus A was seen to have reversal of flow in the umbilical artery and a wave reversal in the ductus venosus. Fetal bradycardia was noted in fetus A. Middle cerebral artery Doppler could not be performed. The couple was informed regarding the facts and the sudden changes in the scan findings a short period of two hours. They were informed regarding the option of laser cord occlusion of the morbid fetus which could be performed as an emergency measure to attempt to save the other fetus.

The couple was naturally confused by the turn of events and wished to be reassessed to see if the condition would improve spontaneously although they were counseled that the only available option would be cord occlusion which was also a difficult procedure with doubtful utility in the present scenario given the sudden deterioration of fetus A. When reassessed after 1 hour, fetus A had undergone cardiac asystole.

The couple was then counseled in detail regarding the possible problems due to co-twin demise in MCDA pair. They understood that the neurological risks to the survivor were high and hence wished to opt for termination of pregnancy. Termination of pregnancy with PGE2 gel was carried out. The patient expelled nine hours after induction.

The findings after expulsion were a surprise as the fetuses had no intervening membrane and had cord entanglement. (Fig. 7) The family refused perinatal examination. However, the clinical photograph when reviewed reveal plethora in one fetus and pallor in the other fetus. (Fig. 8) This suggests that there was an underlying twin-twin transfusion in progress, thus explaining the absent bladder in fetus A and large bladder in fetus B. Unfortunately due to the sudden deterioration of fetus A, middle cerebral artery Doppler evaluation was unavailable to corroborate these findings. The ultrasound images were reviewed and the membrane was seen in both the first trimester and second trimester scans with $\mathrm{T}$ sign and fluid seen on both sides of the membrane.

DISCUSSION: A review of literature and search was made to understand how this was possible. Spontaneous rupture of the inter-twin membrane has been reported in case reports and case series previously and these will be described below.

A case report(1) discussed a known MCDA twin pregnancy where prior ultrasound examination at 11 - 14 weeks, 16 weeks, targeted scan, and 24 weeks previously confirmed "T" sign. The patient was examined at 26 weeks when, no dividing membrane was seen and folded sheets of amnion lay over the placenta. The appearance and adjacency of both twin fetal movements confirmed the diagnosis of spontaneous septostomy of the dividing membrane. (SSDM). The fetuses proceeded 


\section{CASE REPORT}

to 29 weeks and delivered with no complications following preterm labor. No cord entanglement occurred in this case.

Chmait et al(2) put forth criteria to define spontaneous septostomy of the dividing membrane including no prior invasive procedure, absence or disruption of the dividing membrane, both fetuses on the same side of the membrane, umbilical cord entanglement and excessive liquor on both sides of the membrane with other obvious signs of TTTS. Our case had all the features except excessive liquor. Their case series gave a rate as high as $1.8 \%$ but as they are a tertiary referral unit for complicated twin pregnancies, these numbers are unlikely to be representative of the general population.

The most common cause for membrane rupture is iatrogenic either due to a procedure such as amniocentesis or fetal reduction or fetoscopy. Spontaneous septostomy has been reported more commonly in chorio-amnionitis causing weakening of the membrane, excessive fetal movement and polyhydramnios which causes excessive pressures on the dividing membrane. TTTS causes polyhydramnios predisposing to the rupture of membrane. Cases of spontaneous rupture of the membrane, has also been described in dichorionic diamniotic pregnancies ${ }^{(4,5)}$ which has been documented by serial scans.

Most cases reported in literature have reported good outcome with live survival of both fetuses. In our case, the poor outcome was due to the early gestational age, possible twin-twin transfusion syndrome and severe cord entanglement which resulted in death as has been reported previously. ${ }^{(6,7)}$ Entanglement is common in monoamniotic pregnancies and does not always lead to death unless the knots become tight compromising fetal circulation.

In TTTS the septostomy causes equalization of liquor on both sides of the membrane resulting in difficulty in diagnosis (7) as in our case. In some rare instances, the equalization of liquor on both sides of the membrane has resulted in balance of blood flow in the vessels and continuation of the pregnancy ${ }^{(8)}$ with favorable outcome similar to the principle of therapeutic amniotomy which was performed prior to the advent of laser fetoscopy. The other complications of SSDM include pseudomonoamniotic pregnancy, amniotic band syndrome and preterm labor.

CONCLUSION: The learning points from this case were visualization of membrane during one scan does not mean the pregnancy cannot undergo spontaneous rupture of the membrane, regardless of the chorionicity. It is necessary to image the dividing membrane at many areas and not be content with a single image which may mislead. Finally although this is an extremely rare occurrence, awareness of such a condition will greatly facilitate its diagnosis and management.

\section{REFERENCES:}

1. Chadha R, Lange I R, Bratz L. A Rare Case of Antepartum Spontaneous Septostomy in a Monochorionic Diamniotic Twin Pregnancy. Case Reports in Obstetrics and Gynecology Volume 2012, Article ID 748614, doi: 10.1155/2012/748614.

2. Chmait R H, Aghajanian P, Quintero R A et al. Prenatal Diagnosis of Spontaneous of the Dividing Membranes in Complicated Monochorionic Diamniotic Multiple Gestations. J Ultrasound Med 2009; 28:663-668 0278-4297/09.

3. Jeanty C, Newman E, Jeanty P et al. Prenatal Diagnosis of Spontaneous Septostomy in Dichorionic Diamniotic Twins and Review of the Literature. J Ultrasound Med 2010; 29: 455463 0278-4297/10. 


\section{CASE REPORT}

4. R J Abraham. The protective effect of oligohydramnios in a case of missed diagnosis of spontaneous septostomy in monochorionic diamniotic twins. J Obstet Gynaecol. 2013 Feb; 33 (2):205-7. doi: 10.3109/01443615.2012.740530.

5. Reif $\mathrm{P}$, Haeusler $\mathrm{M}$, Gellèn J. Fetal head entrapment through a spontaneous chorionic septostomy in a first trimester dichorionic diamniotic twin pregnancy: a case report. Prenatal Diagnosis Volume 33, Issue 3, pages 298-300, March 2013. DOI: 10.1002/ pd.4061.

6. Fleming T, Miller T. Spontaneous septostomy in monochorionic diamniotic twins resulting in cord entanglement and fetal demise. AJUM August 2012, 15 (3) 103.

7. Bevilacqua E, Aliberti C, D’Ambrosio V. Spontaneous septostomy in monochorionic diamniotic twins: Difficult diagnosis, difficult management. Journal of Obstetrics \& Gynaecology, May 2014, Vol. 34, No. 4: Pages 359-359, (doi: 10.3109/01443615.2013.874408).

8. Nazzaro G, Merenda A, Locci M. Spontaneous septostomy in twin-to-twin transfusion syndrome. A case report. Ultrasound in Obstetrics \& Gynecology 2004; 24: 269-372

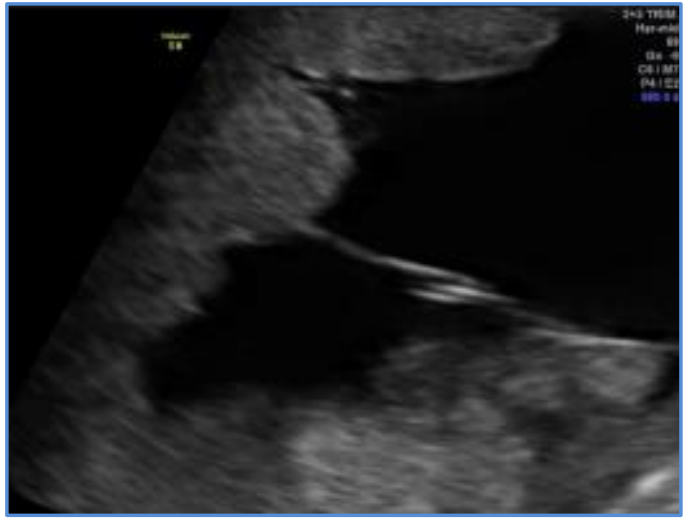

Fig. 1: T Sign

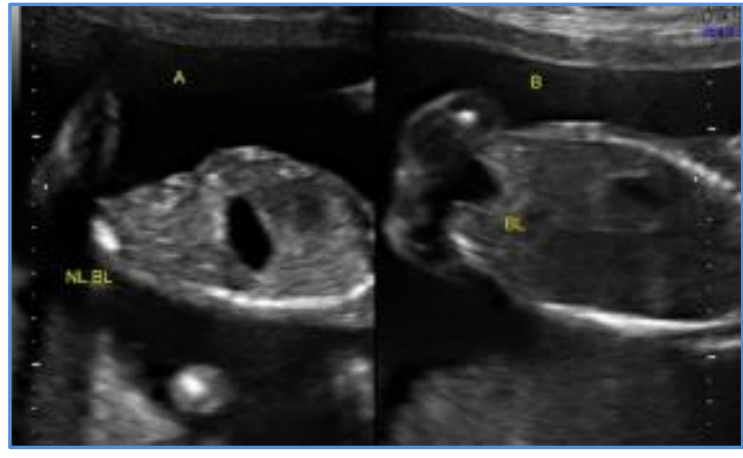

Fig. 3: Bladder discrepancy

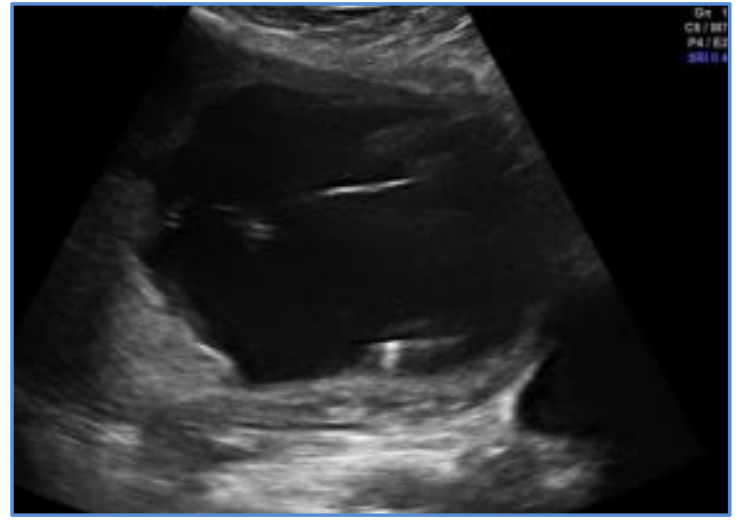

Fig. 2: Inter twin Membrane and liquor

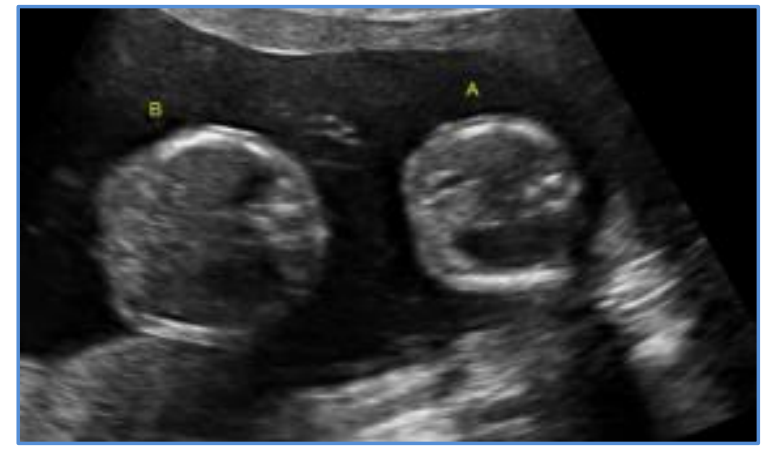

Fig. 4: Growth discrepancy 


\section{CASE REPORT}

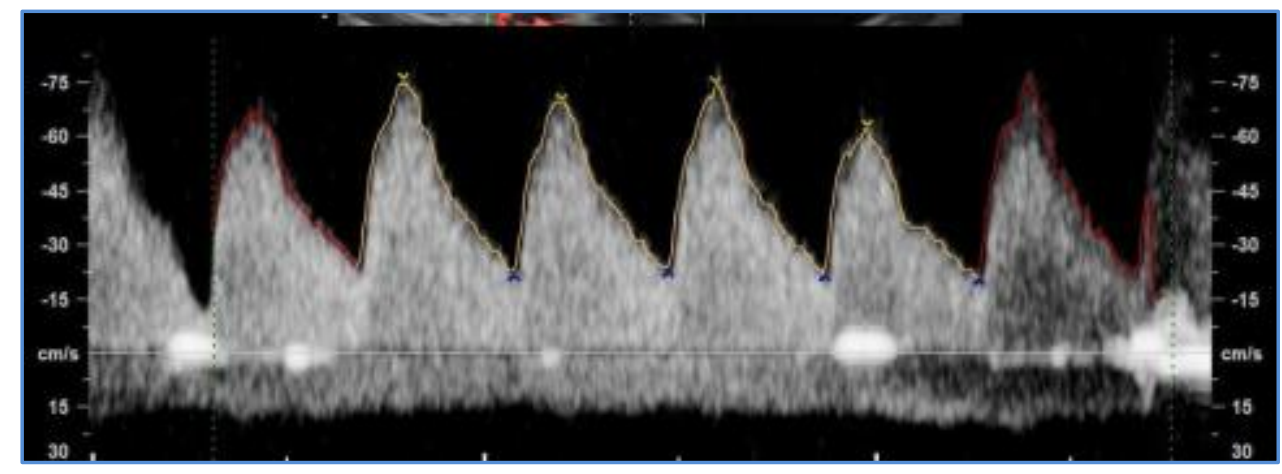

Fig. 5: Normal Umbilical artery Doppler Fetus B

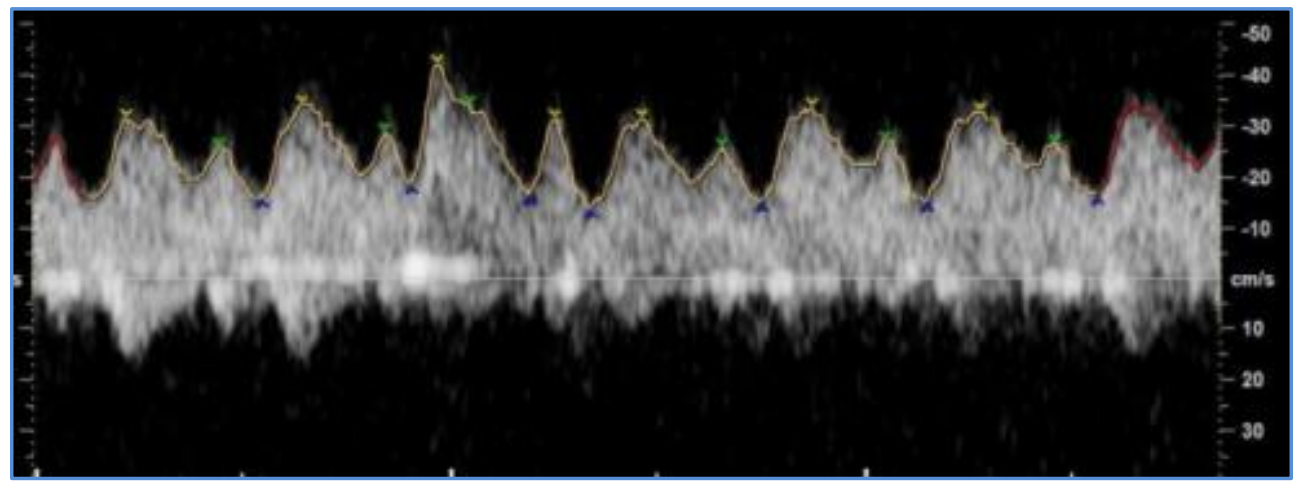

Fig. 6: Normal Ductus venosus Fetus B

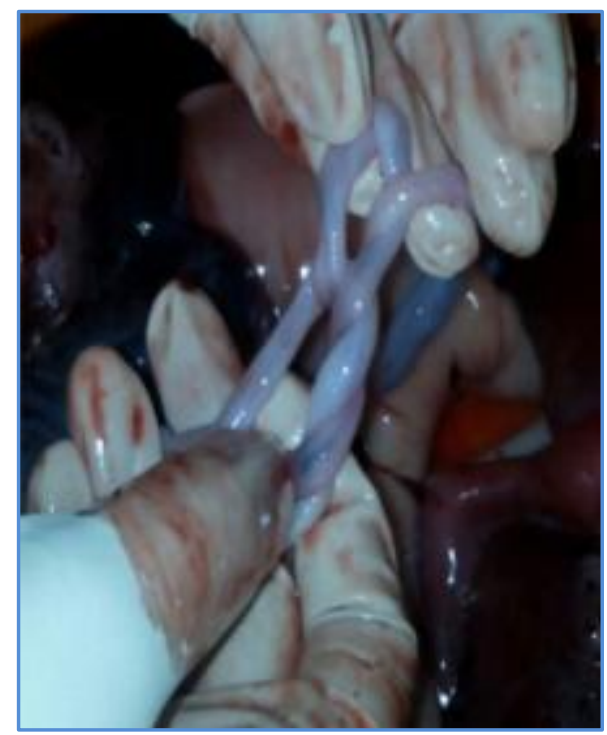

Fig. 7: Cord Entanglement

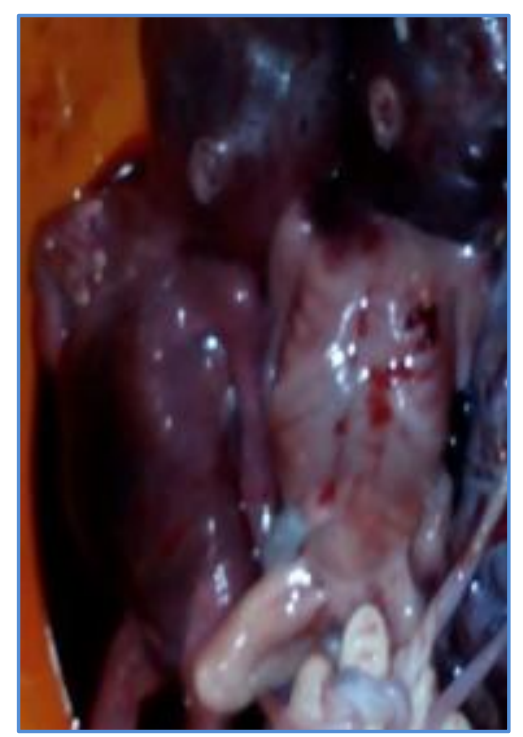

Fig. 8: Fetus A Pallor, Fetus B Plethora 


\section{CASE REPORT}

\section{AUTHORS:}

1. Chitra Andrew

2. K. S. Rajeswari

\section{PARTICULARS OF CONTRIBUTORS:}

1. Associate Professor, Department of Obstetrics and Gynecology, Sri Ramachandra Medical College and Research Institute.

2. Professor, Department of Obstetrics and Gynecology, Sri Ramachandra Medical College and Research Institute.

\section{NAME ADDRESS EMAIL ID OF THE CORRESPONDING AUTHOR:}

Dr. Chitra Andrew, OB-GYN Department, F3 Ward, Sri Ramachandra Medical College and Research Institute, No. 1 Ramachandra Nagar, Porur, Chennai-600116.

Email: chitraandrew@gmail.com

Date of Submission: 04/11/2014. Date of Peer Review: 05/11/2014. Date of Acceptance: 14/11/2014. Date of Publishing: 20/11/2014. 